\title{
microRNA-582 Potentiates Liver and Lung Metastasis of Gastric Carcinoma Cells Through the FOXO3-Mediated PI3K/Akt/Snail Pathway
}

This article was published in the following Dove Press journal: Cancer Management and Research

\author{
Tianyu Xie ${ }^{1,2}$ \\ Di Wu ${ }^{2}$ \\ Shuo $\mathrm{Li}^{1,2}$ \\ Xiongguang $\mathrm{Li}^{1,2}$ \\ Lipeng Wang ${ }^{2}$ \\ Yixun $\mathrm{Lu}^{2}$ \\ Qiying Song ${ }^{2}$ \\ Xuehong Sun ${ }^{2}$ \\ Xinxin Wang ${ }^{1,2}$
}

'School of Medicine, Nankai University, Tianjin 30007I, People's Republic of China; ${ }^{2}$ Department of General Surgery, Chinese PLA General Hospital, Beijing I00853, People's Republic of China
Correspondence: Xinxin Wang $\mathrm{Tel} / \mathrm{Fax}+86-10-66938328$

Email xinxinwangIIII@I63.com
Background: The dysregulation of microRNA (miRNAs) is broadly participated in cancer progression, resulting in sustained cell proliferation by directly targeting various targets. This study investigated the expression of miR-582 in GC and its association with liver metastasis. Methods: Firstly, differentially expressed miRNAs in gastric cancer (GC) tissues were predicted by microarray. Then, the relationship between miR-582 and clinical characteristics of GC patients was analyzed. By silencing of miR-582 in GC cells, the change in malignant biological behaviors of GC cells was detected. The upstream lncRNA, downstream targeting genes of miR-582 and the corresponding signaling pathway were predicted by online databases and verified by luciferase reporter assays, RT-qPCR and Western blot analysis. Finally, the effects of miR-582 on the growth and metastasis of GC cells were detected by in vivo tumorigenesis and metastasis tests.

Results: MiR-582 was highly expressed in GC tissues and related to the metastasis of patients with GC. Silencing of miR-582 expression blocked malignant biological behaviors of GC cells in vitro and in vivo. MiR-582 inhibited forkhead box protein O3 (FOXO3) to upregulate the PI3K/AKT/Snail signaling pathway in GC cells. Besides, GATA6-AS1 was found as an upstream lncRNA to modulate the expression of miR-582.

Conclusion: MiR-582 induced by GATA6-AS1 silencing promotes the growth and metastasis of GC cells by targeting FOXO3 to induce the activation of the PI3K/AKT/Snail signaling pathway. MiR-582 could be a potential molecular therapy target for patients with GC.

Keywords: gastric cancer, miR-582, PI3K/AKT/Snail signaling pathway, proliferation, metastasis, tumorigenesis

\section{Introduction}

Gastric carcinoma (GC) contributes to over $8 \%$ of the total new diagnose and $10 \%$ of total deaths associated with cancer, with more than $70 \%$ of new cases and deaths occurring in under-developed countries. ${ }^{1}$ The risk factors for GC include H. pylori infection, unhealthy diet, smoking history as well as alcohol consumption. ${ }^{2}$ Despite significant progress has been made in surgical treatments and novel chemotherapy protocol development, the overall clinical outcome for advanced GC sufferers is dismal (5-20\% for a 5-year survival and 10 months for a median overall survival). ${ }^{3}$ Epidemiological evidence displays that the liver, peritoneum and lung rank the top three most common sites for metastases, a major cause of poor prognosis of GC. ${ }^{4}$ Additionally, limited metastasis-related biomarkers were substantiated and applied 
in clinic, as a consequence, the detection of new metastasis-related biomarkers for GC is urgently needed for risk prediction and GC metastasis treatment. ${ }^{5}$

MicroRNAs (miRNAs) are a class of non-coding RNAs with 18-25 nucleotides lengthen that repress translation or enhance messenger RNAs (mRNAs) degradation with complementary sequences, and several miRNAs, including miR18a, miR-96, and miR-370 showed elevated expression in GC versus normal or adenoma samples. ${ }^{6}$ Accordingly, our microarray experiments provided data indicating a significant upregulation of miR-582 between GC tissues and paracancerous tissues, highlighting the possible involvement of miR-582 in GC. miR-582 was upregulated in colorectal cancer tissues and cell lines, which was associated with promoted proliferative and migratory ability of the colorectal cancer cells. ${ }^{7}$ Moreover, miR-582-5p suppressed monocyte apoptosis by reducing forkhead box protein O (FOXO) 1 expression and might function importantly in regulating tuberculosis progression. ${ }^{8}$ In the present study, we also predicted and verified a binding site between miR-582 and FOXO3 in GC cells, underscoring the interaction between miR-582 and FOXO3 in GC. Interestingly, the expression of FOXO3a was observed to be substantially lower in cancerous tissues relative to the adjacent non-tumorous tissues, which was tightly linked to lymph node metastasis and unsatisfactory prognosis for GC patients. ${ }^{9}$ In addition, the phosphatidylinositol-3 kinase (PI3K)/protein kinase B (Akt)/ $\beta$-catenin pathway modulated the transcription of Vestigiallike 1 , thus potentiating the proliferation and metastasis in GC. ${ }^{10}$ More importantly, the PI3K/Akt pathway represents one of the most vital pathways interacting with FOXOs in different types of tumors. ${ }^{11}$ miR-582, the most upregulated miRNAs, was selected in the current study to find its binding target genes. Long non-coding RNAs (lncRNAs), a group of transcripts larger than $200 \mathrm{nt}$ in length, exhibit promising values as biomarkers for GC diagnosis and prognosis determination. $^{1-12}$ Moreover, GATA6 antisense RNA 1 (GATA6-AS1) has been indicated to have the highest lung squamous cell carcinoma diagnostic values. ${ }^{2-13}$ However, its connection with miR-582 in GC remains unclear. Therefore, in vivo and in vitro experiments were carried out to explore the effects of the lncRNA/miRNA/target gene network on the $\mathrm{PI} 3 \mathrm{~K} / \mathrm{Akt} /$ Snail pathway, as well as the epithelialmesenchymal transition (EMT), proliferation, migration and invasion of GC cells in vitro and metastasis in vivo. Our findings revealed a novel mechanism regarding miR582 in GC progression.

\section{Patients and Methods}

\section{Patients and Tumor Tissue Samples}

Forty-five malignant GC tissues along with their matched adjacent tissues, with a distance of $>3 \mathrm{~cm} \mathrm{~cm}$ from the GC tissues were attained from patients undergone surgery at the Chinese PLA General Hospital from July 2013 to January 2014. There were 25 males and 20 females (average age $52.62 \pm 10.06$ years). Patients that met the eligibility criteria were enrolled: 1) diagnosed as GC by histopathological examinations; 2) no treatment, including chemotherapy or radiotherapy received before the surgery; 3 ) accessibility of comprehensive clinical data; 4) no previous history of other malignancies or chronic systemic diseases. The clinicopathological information of the $45 \mathrm{GC}$ patients was listed in Supplementary Table 1.

\section{Microarray Experiments and Data Processing}

Six GC tissues were applied to extract $0.5 \mu \mathrm{g}$ total RNA to synthesize cDNA using the GeneChip 3'Invitro Transcription (IVT) Express Kit (902,789, Thermo Fisher Scientific Inc., Waltham, MA, USA) as per the supplier's instructions. Subsequently, cDNA was segmented and hybridized with human miRCURY LNATM Universal RT microRNA PCR Human panel. After hybridization, the arrays were scanned with GeneChipTM Scanner 3000 7G system (000213, Thermo Fisher Scientific).

\section{Reverse Transcriptase Polymerase Chain Reaction and Quantitative Real-Time PCR (RT-qPCR)}

The total RNA was isolated from tissues and cells with the application of TRIzol reagent (Invitrogen, Carlsbad, CA, USA). The primers used in the experiment including GATA6-AS1, miR-582, U6, FOXO3 and GAPDH were from Applied Biosystems (ABI, Foster City, CA, USA). ABI 7500 was utilized to performed the qPCR work.

\section{Cell Culture and Treatment}

The GES-1 (immortalized gastric mucosa epithelial cells) and the BGC-823, MKN-45, HGC-27, SGC-7901 and MGC-803 (GC cells) were all purchased from Cell Bank of Shanghai Institute of Cells, Chinese Academy of Science (Shanghai, China). After being plated in cell culture dishes at $1 \times 10^{5}$ cells $/ \mathrm{cm}^{2}$, the cells were grown in Dulbecco's modified Eagle's medium or Roswell Park 
Memorial Institute-1640 medium containing 10\% fetal bovine serum (Gibco, Grand Island, NY, USA) for 48 h with $5 \% \mathrm{CO}_{2}$ at a temperature of $37^{\circ} \mathrm{C}$. When cells were covered with $80-90 \%$ microscopic view, the trypsinized cells were sub-cultured.

The BGC823 cells and MKN-45 cells with good growth were transfected with the synthesized miR-582 inhibitor and the corresponding inhibitor control, respectively. The Lipofectamine ${ }^{\mathrm{TM}} 3000$ system (Invitrogen, Carlsbad, CA, USA) was applied for viral transfection.

\section{Cell Viability Assays}

The viabilities of cells were examined by 5 '-ethynyl-2'deoxyuridine (EdU) staining as described by Yang et al. ${ }^{14}$ The cell proliferation activity was detected using an EdU staining proliferation kit (ab222421, Abcam). In general, cells in good growth conditions were incubated for $2 \mathrm{~h}$ at $37^{\circ} \mathrm{C}$ with the staining solution in the kit. After counterstaining of the nucleus with 4',6-Diamidino2-Phenylindole (DAPI), the number of EdU positive cells in each well was counted in three different visual fields. The apoptosis level of GC cells was detected by Hoechst 33,258 staining as previously reported. ${ }^{15}$ In short, apoptotic cells in each well were counted in three different visual fields. The number of apoptotic cells in each field was divided by the total number of cells in field as the apoptotic rate.

\section{Immunofluorescence Staining}

The expression of EMT markers in GC cells was detected by immunofluorescence. The frozen sections $(5-\mu \mathrm{m}$ in thickness) were fixed in acetone and sealed with $1 \%$ bovine serum albumin (BSA) in the phosphate buffered saline (PBS). The antibodies against N-cadherin (1:1000, ab18203, Abcam Inc., Cambridge, UK) and E-cadherin (1:100, ab48394, Abcam) were used for an overnight staining at $4{ }^{\circ} \mathrm{C}$. The secondary antibody to rabbit (1:5000, ab150088, Abcam) labeled with fluorescein isothiocyanate was incubated for $1 \mathrm{~h}$ at $37^{\circ} \mathrm{C}$. Following blocking endogenous biotin using a streptavidin-biotin blocking kit (Vector Laboratories, Inc., Burlingame, CA, USA), the cells were then counter-stained with DAPI and visualized with a fluorescence microscope (Leica DM 3000, Leica Microsystems $\mathrm{GmbH}$, Wetzlar, Germany).

\section{Transwell Assay for Migration and Invasion}

Cell invasion and migration were tested by Transwell assay in strict accordance with the previous literature. ${ }^{16}$ The apical chamber of the Transwell plate was coated with $50 \mu \mathrm{L}$ Matrigel (BD Biosciences, Franklin Lakes, NJ, USA). The cells $\left(5 \times 10^{4}\right)$ were suspended in $100 \mu \mathrm{L}$ serum-free medium and then seeded into the upper layer of the Transwell chamber (BD Biosciences). Totally 500 $\mu \mathrm{L}$ medium containing $20 \%$ fetal bovine serum was added to the basolateral chamber. After $48 \mathrm{~h}$ at $37^{\circ} \mathrm{C}$ with $5 \%$ $\mathrm{CO}_{2}$, cells retained in the apical chambers were scratched. The cells in the basolateral chamber were fixed in methanol, stained in $0.1 \%$ crystal violet and photographed under an inverted contrast microscope (CK2, Olympus, Japan). For the migration experiment, the apical chamber was free of Matrigel, and the remaining operations were consistent with the invasion experiment.

\section{Luciferase Reporter Assays}

The 3'untranslated region (UTR) binding sequences of miR-582 and GATA6-AS1 as well as FOXO3 were predicted by online prediction softwares RNA22, StarBase and DIANA. FOXO3 or GATA6-AS1 wild type (WT) and 3'UTR binding sequence mutant (MT) were synthesized by a commercial company Shanghai Sangon Biological Engineering Technology \& Services Co., Ltd. (Shanghai, China) and inserted into pMIR-REPORT ${ }^{\mathrm{TM}}$ (Thermo Fisher Scientific). The engineered luciferase reporter plasmids were delivered with miR-582 mimic or miRNA NC into $293 \mathrm{~T}$ cells using the Lipofectamine ${ }^{\mathrm{TM}}$ 3000 kit (Invitrogen) following the supplier's instructions. The relative luciferase activity was evaluated after 24 $\mathrm{h}$ with the Dual-Luciferase Reporter Assay System (Promega Corporation, Madison, WI, USA).

\section{Immunoblotting Assay}

The cells were lysed on ice in radio immunoprecipitation assay lysis buffer containing a mixture of proteinase and phosphatase inhibitors for $30 \mathrm{~min}$ and then centrifuged at $13,000 \mathrm{~g}$ for $10 \mathrm{~min}$. The protein concentration was subsequently measured using a bicinchoninic acid kit. A total of 20 $\mu \mathrm{g}$ protein was separated by $10 \%$ sodium dodecyl sulfatepolyacrylamide gel electrophoresis and then transferred onto a polyvinylidene difluoride membrane. Subsequently, the membranes were blocked with 5\% BSA and incubated with the diluted primary antibodies against $\mathrm{pFOXO} 3^{\mathrm{S} 253}$ (1:2000, 
ab47285, Abcam), PI3K (1:5000, ab1549, Abcam), pPI3K $^{\mathrm{Y} 607}$ (1:5000, ab182654, Abcam), AKT1 (1:5000, ab235958, Abcam), pAKT1 ${ }^{\mathrm{S} 473}$ (1:50, ab812, Abcam) and Snail $\left(1: 50\right.$, ab53519, Abcam) at $4^{\circ} \mathrm{C}$ overnight. After 3 rinses with Tris-buffered saline Tween-20 (TBST), the membranes were incubated with the horseradish peroxidase (HRP)-conjugated goat anti-rabbit secondary antibody (1:10,000, ab7090, Abcam) for $1 \mathrm{~h}$. After 3 rinses with TBST, the membranes were visualized using a SuperSignal West Pico Kit (Thermo Fisher Scientific Inc). Images were captured using the ChemiDoc ${ }^{\mathrm{TM}}$ XRS (Bio-Rad, Inc., Hercules, CA, USA). $\beta$-actin was regarded as the loading control. The expression of target protein $=$ gray value of target protein bands/gray value of $\beta$-actin.

\section{Mice Experiments}

In total, 24 mice (aged 4-6 weeks; weight, $20 \pm 2 \mathrm{~g}$, Beijing Vital River Laboratory Animal Technology Co., Ltd., Beijing, China) were used. HepG2 and SMMC7721 cells $\left(4 \times 10^{6}\right)$ transfected with miR-582 inhibitor or mock in $2 \mathrm{~mL}$ saline were injected into BALB/c nude mice subcutaneously $(\mathrm{n}=6)$. After $7 \mathrm{~d}$ of injection, the tumor size was examined every $7 \mathrm{~d}$ for $28 \mathrm{~d}$ totally. Tumor volume $(\mathrm{V})$ was evaluated by measuring the length $\left(\mathrm{m}_{2}\right)$ and width $\left(\mathrm{m}_{1}\right)$ with caliper and calculated with the formula $\mathrm{V}=\mathrm{m}_{1}{ }^{2} \times \mathrm{m}_{2} \times 0.5236$. By the end of the experiment, the nude mice were euthanized with overdose of pelltobarbitalum natricum at $120 \mathrm{mg} / \mathrm{kg}$. The tumor was harvested for histological examination.

\section{Immunochemistry}

The number of KI67 positive cells in tumor sections was detected using immunohistochemistry as previously reported. ${ }^{17}$ Briefly, tumor tissue sections were deparaffinized, rehydrated, and then incubated at $95^{\circ} \mathrm{C}$ in citrate buffer for $20 \mathrm{~min}$ for antigen retrieval. At room temperature, nonspecific binding was blocked with $10 \%$ normal goat serum for $30 \mathrm{~min}$. The sections were cultured with the primary antibody to $\operatorname{KI67}(1: 1000$, ab15580; Abcam) at $4^{\circ} \mathrm{C}$ overnight and with HRP-conjugated goat anti-rabbit secondary antibody to $\operatorname{IgG}(1: 500 ; \mathrm{ab} 6721 ; \mathrm{Abcam})$ at $30^{\circ} \mathrm{C}$ for $30 \mathrm{~min}$. At room temperature, the sections were then reacted with the peroxidase coupled with the antibiotic streptomycin at room temperature for $30 \mathrm{~min}$. The reaction products were visualized using a diaminobenzidine kit (Beijing Solabio Life Sciences Co., Ltd., Beijing, China) and quantitatively analyzed by NIH Image $J$ software (Bethesda, MD, USA).

\section{Tail Vein Injections into Nude Mice}

The nude mice were injected with cells $\left(4 \times 10^{6}\right)$ stably transfected with miR-582 inhibitor or mock via the tail veins, which were euthanized 30 or $45 \mathrm{~d}$ after injection with pelltobarbitalum natricum. The lungs and liver were removed to conduct the hematoxylin and eosin (HE) staining for further analysis.

\section{Statistics}

The results were all analyzed using SPSS version 21.0 software (SPSS, Inc., Chicago, IL, USA). The normal distribution of data was tested using KolmogorovSmirnov and represented by mean \pm standard deviation (SD). Pairwise differences between two groups were analyzed using unpaired $t$-test. One-way or two-way analysis of variance (ANOVA) with Tukey's post hoc test were used for evaluating the difference among multiple groups. Fisher's exact test was applied to analyze the association of enumeration data. Data were considered statistically significant if $p$ value less than 0.05 .

\section{Results}

\section{The High Expression of miR-582 in GC Tissues Is Related to Metastasis in GC}

First, we used the microRNA microarray to detect the cancer tissues and adjacent normal tissues from 5 patients with GC. By using log FoldChange $>2$, adjust $\mathrm{p}<0.05$ as the screening condition, we screened out 695 differentially expressed miRNAs, of which 396 were up-regulated and 299 were down-regulated. The heatmap shows the top 30 differentially expressed miRNAs in Figure 1A. Then, we detected the expression of miR-582, miR-493, miR-338, miR-1254, miR-34a and miR-654 in $45 \mathrm{GC}$ tissues and paracancerous tissues by RT-qPCR, and observed that miR-582 was the most enhanced one in GC tissues (Figure 1B). Also, we monitored that the miR-582 expression in tissues from GC patients with liver and lung metastases was remarkably promoted versus GC patients free of liver, lung and lymph node metastases (Figure 1C-E). Subsequently, we tested the expression of miR-582 in GC cell lines and GES- 1 cells, and found that the miR-582 was drastically enhanced in the $\mathrm{GC}$ cell lines, and the promotion of that was more pronounced in BGC-823 and MKN-45 cells (Figure 1F). Therefore, in order to verify the effect of miR-582 on the GC cell malignant potentials, we transfected miR-582 inhibitor into BGC-823 and MKN-45 


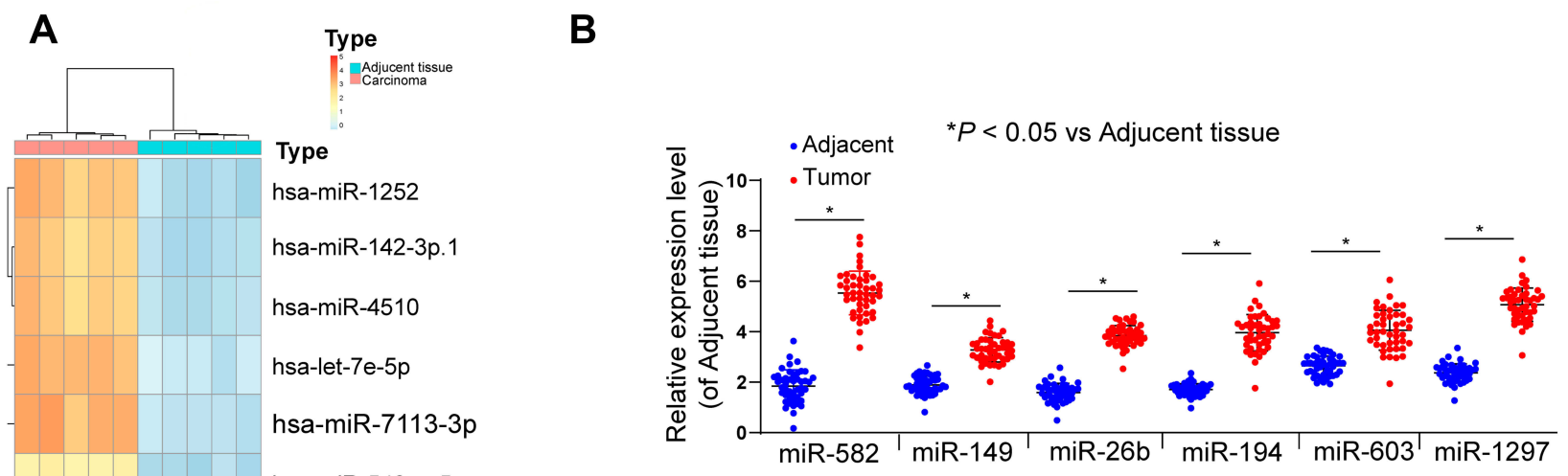

hsa-miR-548az-5p

hsa-miR-4319

hsa-miR-6869-5p C

C D

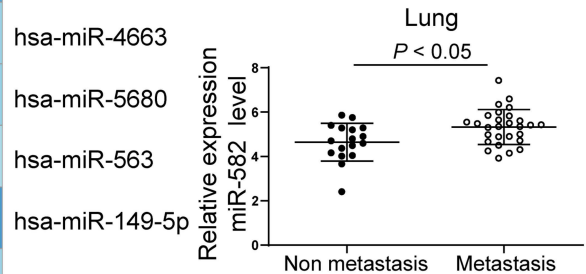

hsa-miR-1297

hsa-miR-26b-5p

hsa-miR-582-5p

F

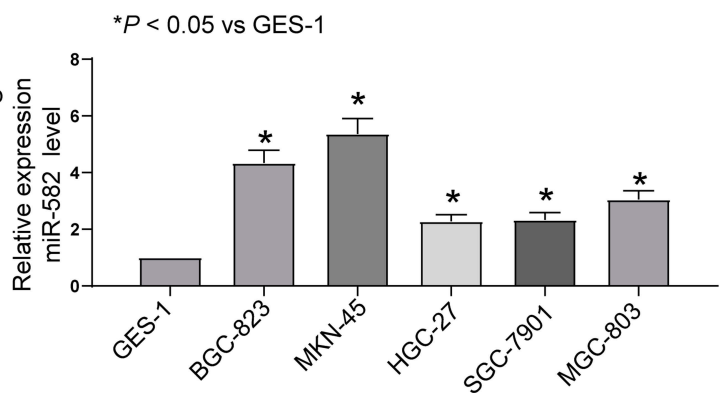

E

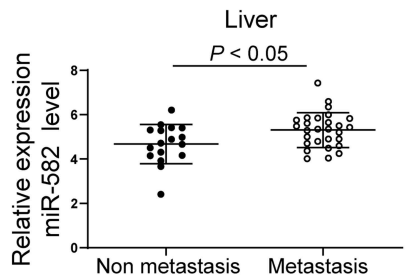

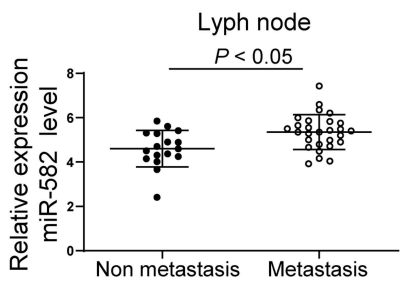

G

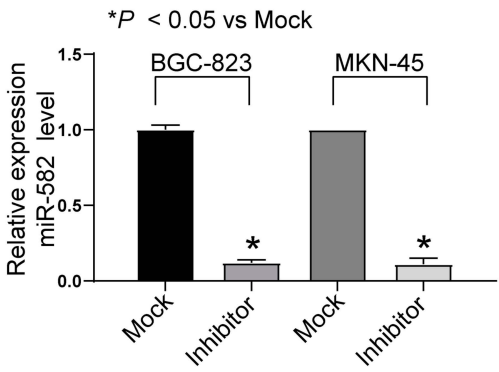

Figure I miR-582 is increased in GC tissues and linked to metastasis. (A) microarray was performed to determine dysregulated miRNAs using miRCURY LNA ${ }^{\text {TM }}$ Universal RT microRNA PCR Human panel. (B) The miR-582, miR-149, miR-26b, miR-194, miR-603 and miR-1297 expression in adjacent and tumor tissues of 45 GC patients evaluated by RT-qPCR. (C) The miR-582 expression in 45 GC patients diagnosed with lung metastasis evaluated by RT-qPCR. (D) The miR-582 expression in 45 GC patients diagnosed with liver metastases evaluated by RT-qPCR. (E) The miR-582 expression in 45 GC patients diagnosed with lyph node metastases evaluated by RT-qPCR. (F) The miR-582 expression in GES-I and GC cell lines evaluated by RT-qPCR. (G) miR-582 expression in BGC-823 and MKN-45 cells treated with miR-582 inhibitor and inhibitor control evaluated by RT-qPCR. In panel (B and F), one-way ANOVA and Tukey's multiple comparisons test was applied to determine statistical significance, while in panel (C-E), unpaired $t$-test was used. $*_{p}<0.05$ vs inhibitor control (Mock).

cells. The transfection efficiency was tested by RTqPCR (Figure 1G). Together, our findings show that miR-582 is promoted in GC tissues and cell lines.

\section{miR-582 Enhances the Malignant Potentials of GC Cells}

The results of EdU assay and Hoechst 33,257 staining exhibited that inhibition of miR-582 expression reduced cell proliferation and viability and even promoted apoptosis (Figure 2A and B). The immunofluorescence staining of EMT makers E-cadherin and N-cadherin demonstrated that the decrease of miR-582 weakened the EMT of GC cells (Figure 2C). Then, we carried out Transwell experiments to test the cell invasion and migration (Figure 2D and E), and it was found that the BGC-823 and MKN-45 cell migration and invasion were inhibited after the treatment with miR-582 inhibitor. These data propose that miR-582 inhibitor diminishes the proliferation, invasion, migration and EMT abilities of the GC cells. 


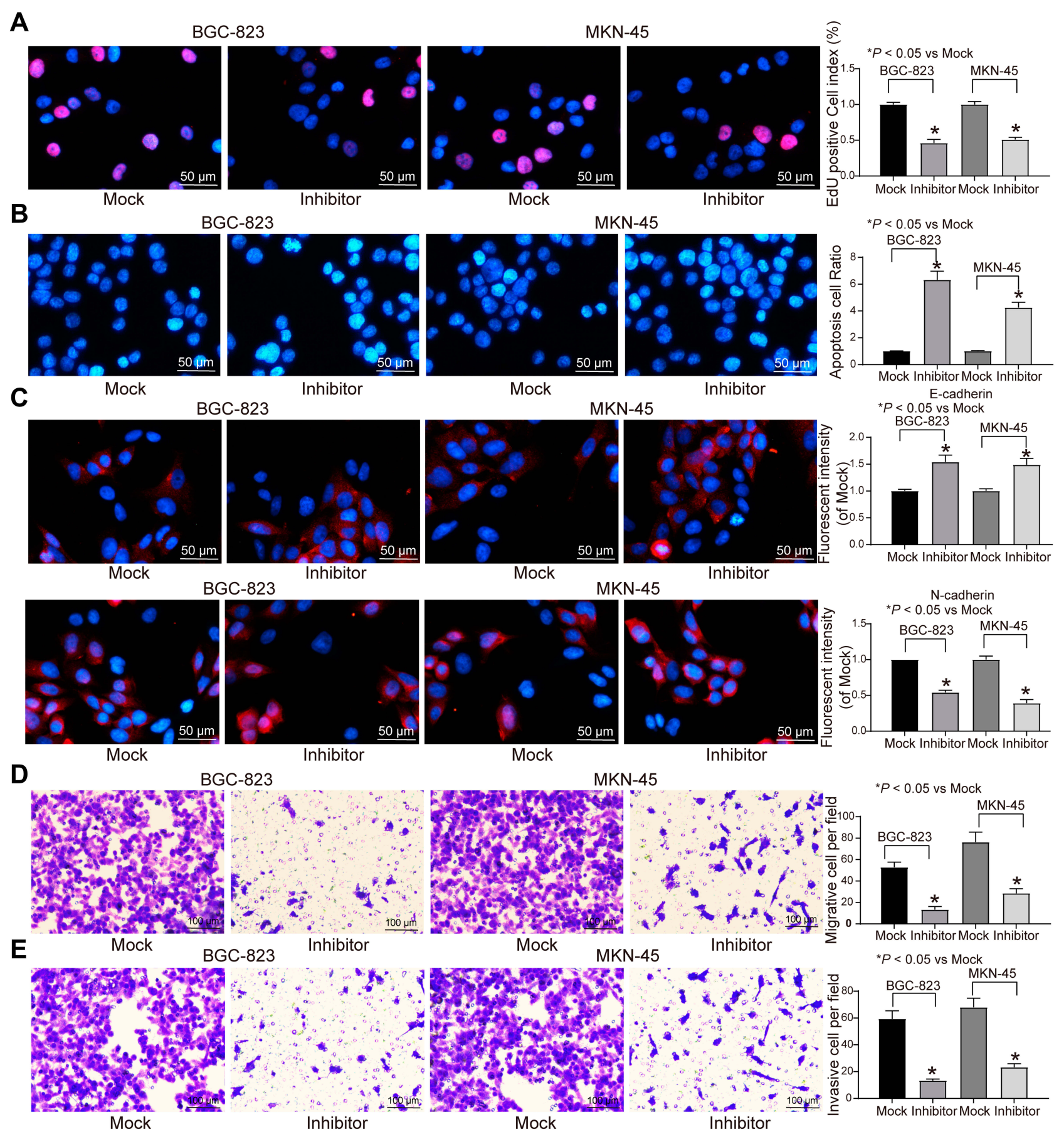

Figure 2 miR-582 inhibitor attenuates GC cell malignant behaviors. (A) EdU staining of GC cell viability. (B) Hoechst 33,258 staining of GC cell viability. (C) Immunofluorescence of E-cadherin and N-cadherin. (D) Migration ability of GC cells examined by Transwell assays. (E) Invasion ability of GC cells assessed by Transwell assays. The data are displayed as the mean \pm SD of three independent experiments. One-way ANOVA and Tukey's multiple comparison test was applied to determine statistical significance. ${ }^{*} p<0.05$.

\section{miR-582 Facilitates Malignant Phenotypes in $\mathrm{GC}$ by Binding to FOXO3}

In order to clarify the downstream mechanism of miR-582, the target genes of miR-582 were predicted and screened out by StarBase, TargetScan and miRSearch. We screened out a large number of genes, in which $\mathrm{FOXO} 3$ drew our attention. Furthermore, miR-96-5p can target FOXO3 to promote the growth of GC cells. ${ }^{18}$ Thus, we examined FOXO3 expression in cancer tissues of 45 GC sufferers (Figure 3A). FOXO3 was suggested to be significantly downregulated and negatively correlated with miR-582 expression (Figure 3B). Subsequently, we found that the 
FOXO3 expression in cancer tissues of GC patients with lung and liver metastases was markedly reduced relative to GC patients without liver, lung and lymph node metastases (Figure 3C-E).

Therefore, the target binding relationship between miR-582 and FOXO3 was detected by luciferase report assays (Figure 3F). Besides, miR-582 could target FOXO3 directly in GC cells, as the FOXO3 expression at both mRNA and protein levels in BGC-823 and MKN-45 cells found to be increased by miR-582 inhibitor (Figure $3 \mathrm{G}$ and $\mathrm{H}$ ). These data imply that miR-582 induces the progression of GC by interacting with FOXO3.

\section{miR-582 Promotes Invasion and Migration of GC Cells by Activating the PI3K/Akt/Snail Pathway Through FOXO3}

A previous study pointed out that FAM83A induced the EMT of non-small cell lung cancer by potentiating the $\mathrm{PI} 3 \mathrm{~K} / \mathrm{Akt} /$ Snail pathway, thereby promoting the metastasis of lung cancer. ${ }^{19}$ Thus, we used immunoblotting to detect the protein expression of PI3K/Akt/Snail and the extent of $\mathrm{PI} 3 \mathrm{~K} / \mathrm{Akt} /$ Snail phosphorylation in BGC-823 and MKN-
45 cells treated with miR-582 inhibitor or mock. As shown Figure 4A, pPI3K and pAKT1 were significantly inactivated in miR-582 inhibitor-treated GC cells.

Furthermore, in order to verify the role of the PI3K/ Akt/Snail pathway in miR-582-mediated proliferation and metastasis, we used a PI3K-specific agonist P740Y-P on miR-582 inhibitor-transfected cells. Decreased viability, EMT, migration and invasion of BGC-823 and MKN-45 cells and increased apoptosis by miR-582 inhibitor were reversed by P740Y-P (Figure 4B-E).

\section{miR-582 Potentiates Tumor Growth and Metastasis in vivo}

To validate the effects of miR-582 in vivo, BGC-823 and MKN-45 cells treated with mock or miR-582 inhibitor were subcutaneously injected into nude mice. The tumor volume growth curve and the weight columns revealed that growth rate of the GC cells was slowed by miR-582 inhibitor (Figure 5A and B). Subsequently, we used the immunohistochemistry to detect the number of KI67 positive cells in the tumor, and we found that the downregulation of miR-582 repressed the rate of positive cells in the tumor (Figure 5C).
A

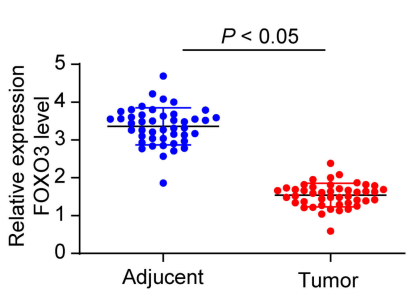

E

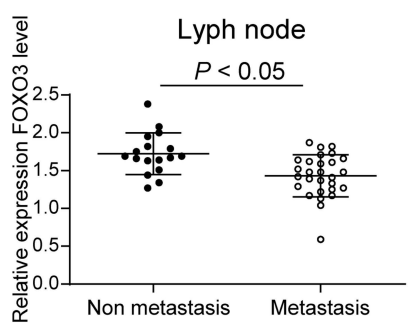

B

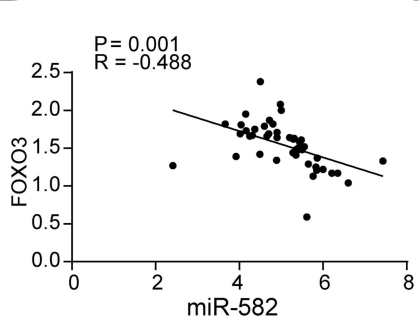

$\mathbf{F}$

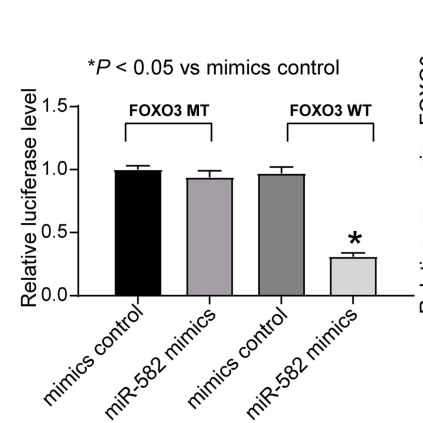

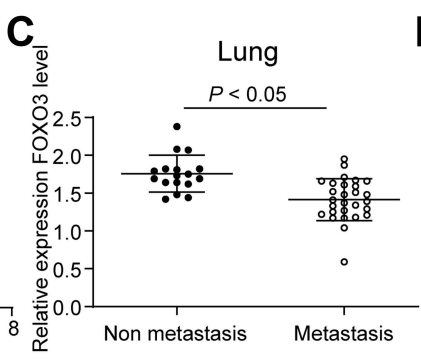

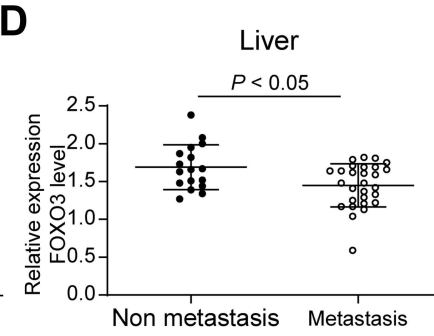

G

\section{H}
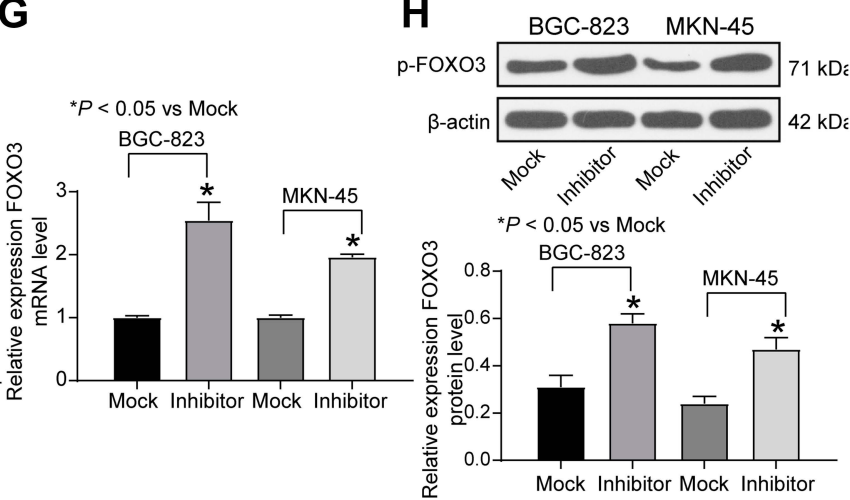

$\beta$-actin
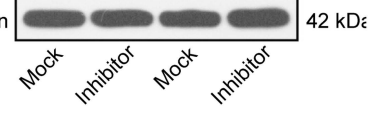

${ }^{*} P<0.05$ vs Mock

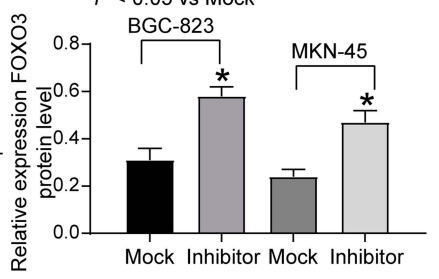

Figure 3 miR-582 promotes GC cell malignant behaviors by targeting FOXO3. (A) FOXO3 expression in adjacent and tumor tissues of 45 GC patients examined by RTqPCR. (B) The correlation analysis between miR-582 expression and mRNA expression of FOXO3 by Pearson's correlation test. (C) The mRNA expression of FOXO3 in $45 \mathrm{GC}$ patients diagnosed with lung metastasis. (D) The mRNA expression of FOXO3 in $45 \mathrm{GC}$ patients diagnosed with liver metastasis. (E) The mRNA expression of FOXO3 in 45 GC patients diagnosed with lyph node metastases evaluated by RT-qPCR.(F) The combination of miR-582 and FOXO3 verified by dual luciferase reporter assays. (G) The mRNA expression of FOXO3 in BGC-823 and MKN-45 cells treated with miR-582 inhibitor or mock determined by RT-qPCR. (H) The protein expression of FOXO3 in BGC-823 and MKN-45 cells treated with miR-582 inhibitor or mock determined by immunoblotting. The data are displayed as the mean \pm SD of three independent experiments. One-way ANOVA and Tukey's multiple comparison test was applied to determine statistical significance. ${ }^{*} p<0.05$. 

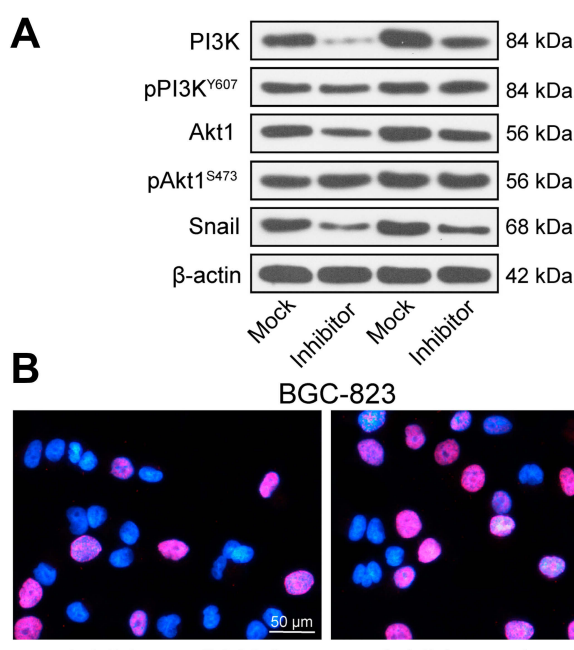

c

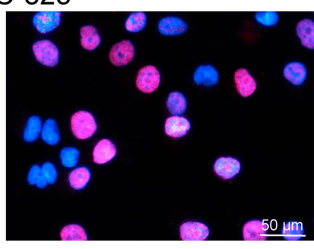

Inhibitor + Agonist
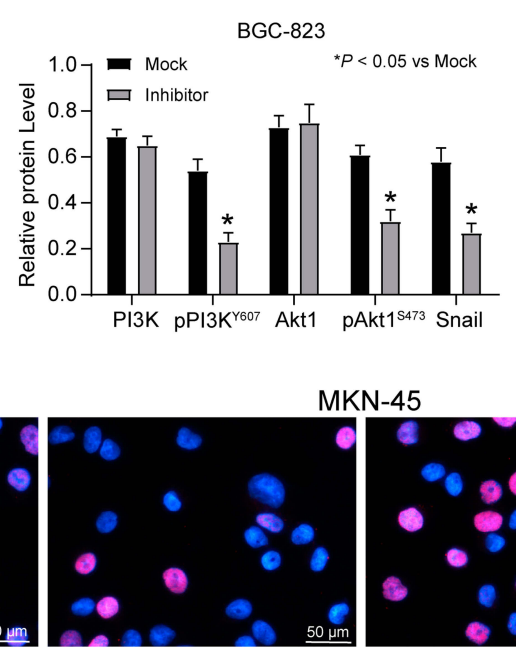

Inhibitor + DMSO
MKN-45

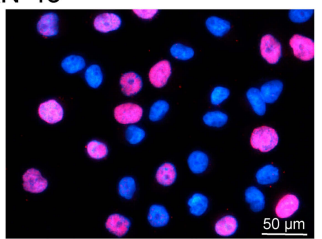

Inhibitor + Agonist

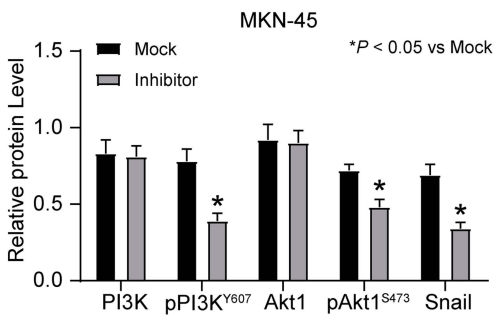

${ }^{*} P<0.05$ vs Inhibitor + DMSO

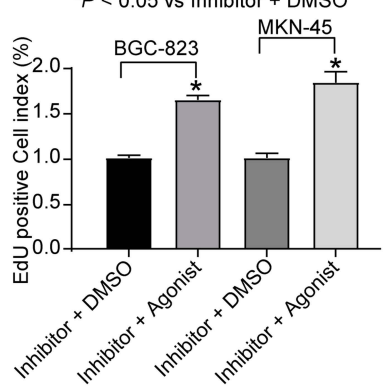

MKN-45

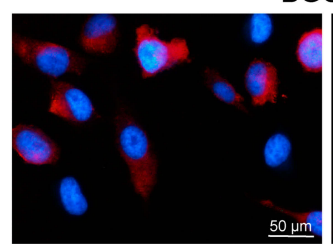

Inhibitor + DMSO

BGC-823

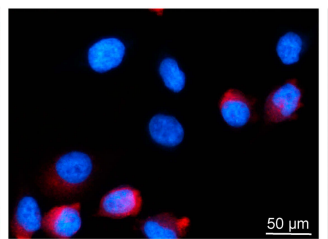

Inhibitor + Agonist

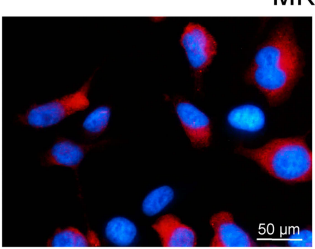

Inhibitor + DMSO

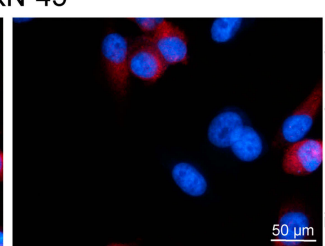

Inhibitor + Agonist

D
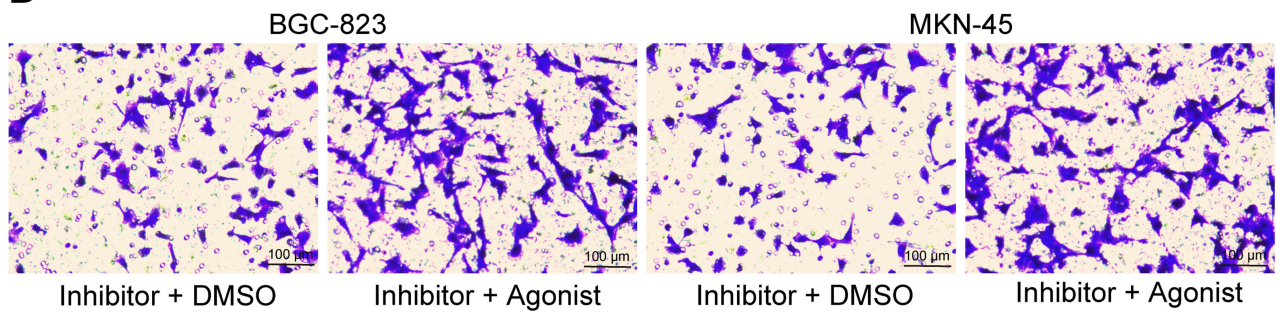

MKN-45
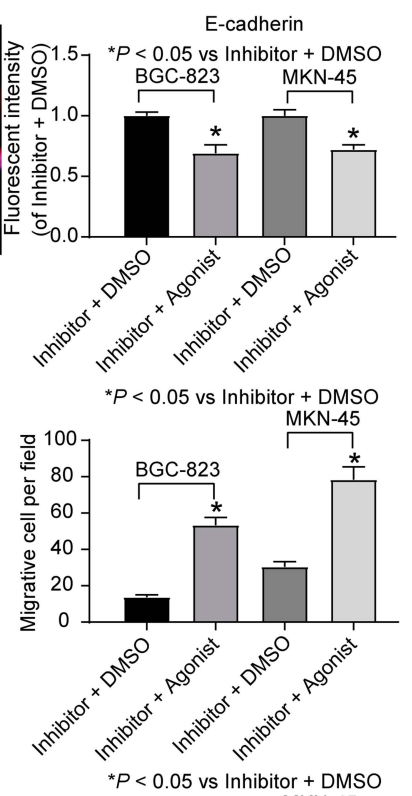

E
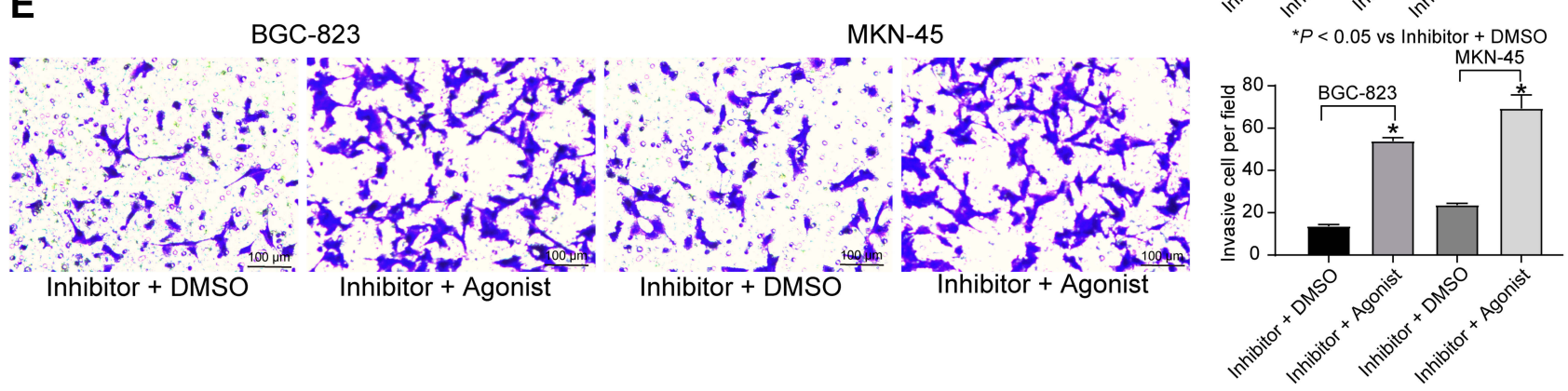

Figure 4 miR-582 activates the PI3K/Akt/Snail pathway in GC cells. (A) The protein expression and phosphorylation level of PI3K/Akt/Snail signaling pathway measured by immunoblotting. Then, PI3K specific agonist 740Y-P was added into miR-582 inhibitor-transfected cells. (B) EdU staining of GC cell viability. (C) immunofluorescence of E-cadherin. (D) Migration ability of GC cells assessed by Transwell assays. (E) Invasion ability of GC cells evaluated by Transwell assays. The data are displayed as the mean \pm SD of three independent experiments. One-way ANOVA and Tukey's multiple comparison test was applied to determine statistical significance. $*_{p}<0.05$.

To directly verify the role of miR-582 in metastasis in vivo, we conducted a separate analysis in nude mice by injecting stable miR-582-knocked down BGC-823 and MKN-45 cells in the tail vein. The mice injected with stable miR-582depleted cells had markedly fewer metastases in lung and liver than that of mice injected with stable mock-depleted 
cells (Figure 5D). The promotive effect of miR-582 on lung and liver metastasis of GC was further confirmed in vivo.

\section{GATA6-ASI Regulates miR-582 Expression in GC Cells}

We subsequently screened five lncRNAs on the upstream of miR-582, NEAT1, KCNQ1OT1, MIR17HG, GATA6-AS1 as well as PRKCQ-AS1 (Figure 6A) based on RNA22, StarBase and DIANA bioinformatics prediction websites. Moreover, we predicted the expression of the five lncRNAs in stomach adenocarcinoma (STAD) as well as in normal tissues through GEPIA websites. We observed that only NEAT1 and GATA6AS1 had significant differences (Figure 6B). GATA6-AS1 overexpression has been reported in a literature to significantly inhibit GC proliferation and EMT. $^{3-20}$ As a consequence, we used RT-qPCR to detect the expression of GATA6-AS1 in tumor tissues and paracancerous tissues in 45 GC patients, and found that GATA6-AS1 was significantly lower in GC tissues (Figure 6C). We subsequently verified the binding relationship between GATA6-AS1 and miR-582 by dual luciferase experiments. The luciferase activity in 293T cells decreased significantly after transfection of GATA6-AS1-WT and miR-582 mimic (Figure 6D). Given the aforementioned findings, we concluded that GATA6-AS1 negatively regulated the miR-582 expression in GC.

\section{Discussion}

Comparable to other cancers, gene expression patterns are altered in GC, one of the most frequent cancers throughout the world, and two main categories of genes are
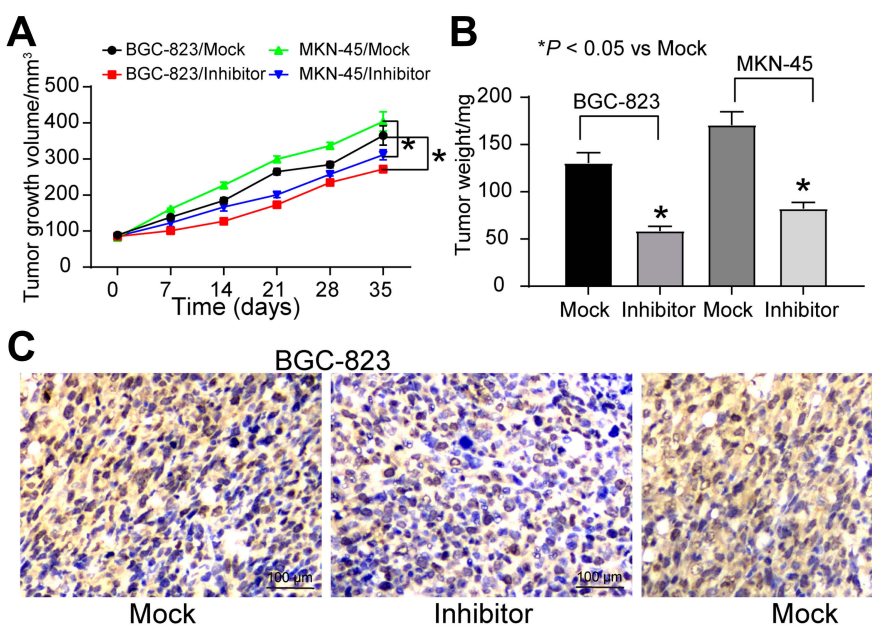

GC-823

$$
\text { Mock Inhibitor Mock Inhibitor }
$$
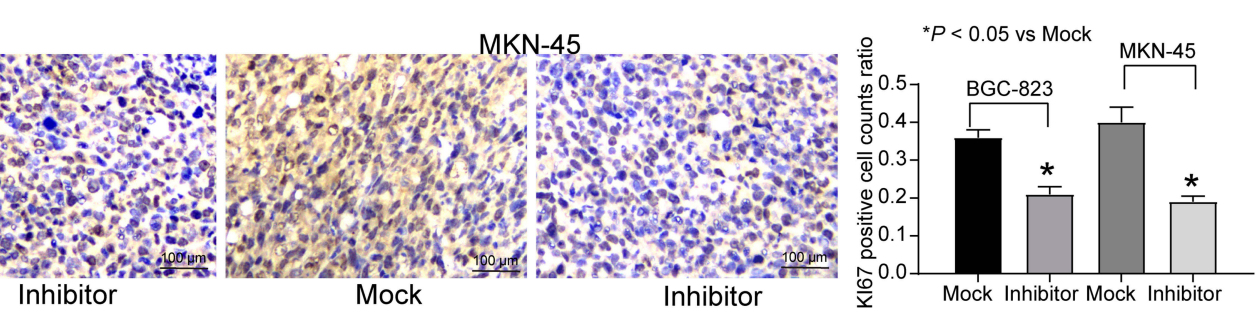

D

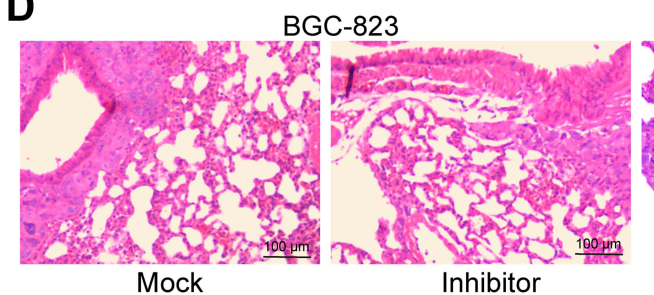

BGC-823

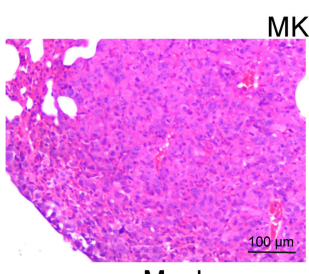

$\mathrm{MKN}-45$
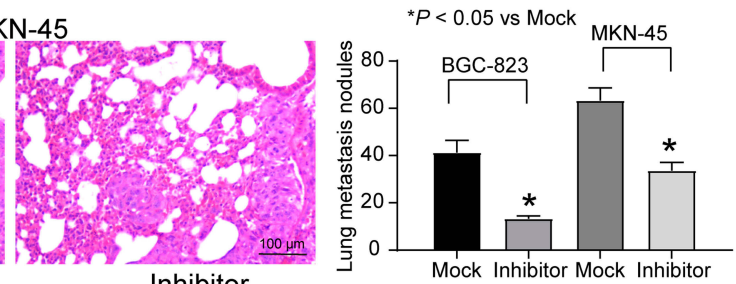

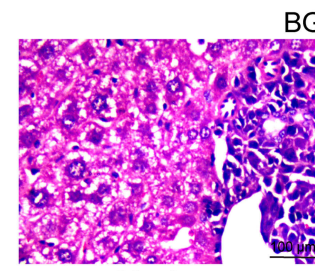

Mock
BGC-823

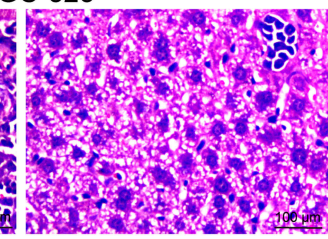

Inhibitor

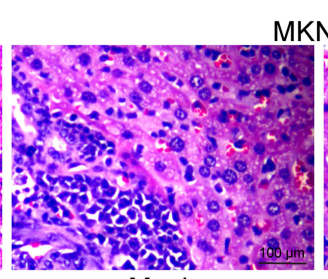

Mock
MKN-45

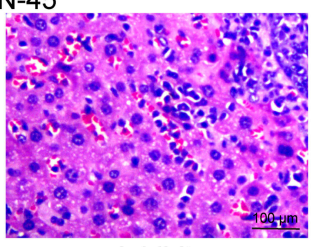

Inhibitor

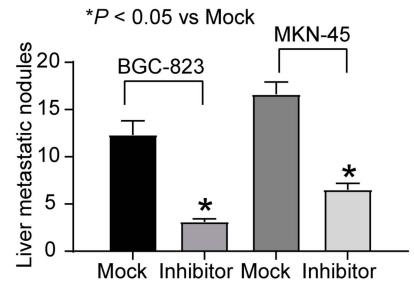

Figure 5 miR-582 inhibitor reduces GC cells growth and metastasis in vivo. BGC-823 and MKN-45 cells transfected with miR-582 inhibitor or inhibitor control were injected subcutaneously into BALB/c nude mice at $5 \times 10^{6}$ each mouse $(n=6)$. Tumor growth was measured continuously every $7 \mathrm{~d}$. Thirty-five days after injection, the mice were euthanized by overdosed pelltobarbitalum natricum. (A) Tumor size. (B) Tumor weight. (C) Immunostaining of Ki67-positive tumor cells and quantification. (D) HE staining of lung and liver metastases in nude mice injected with BGC-823 cells by tail-vein. The numbers of metastasis were determined at 30 days after BGC-823 cells injection or 30 days after MKN-45 cells injection. One-way or two-way ANOVA and Tukey's multiple comparison test was applied to determine statistical significance, ${ }^{*} p<$ 0.05 . 


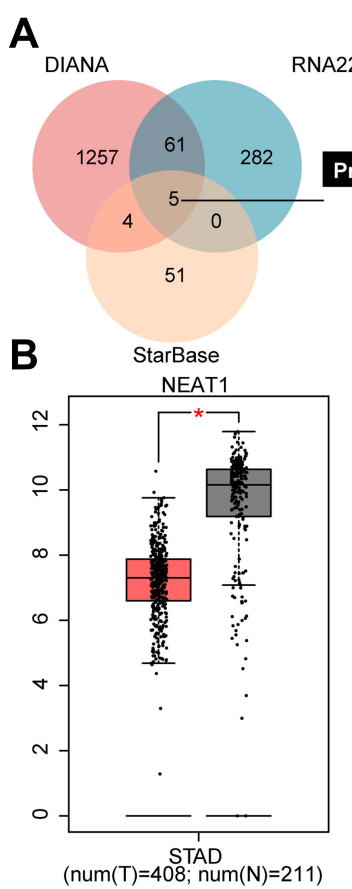

C

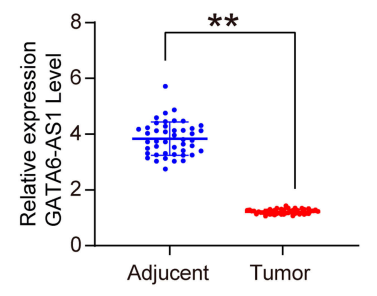

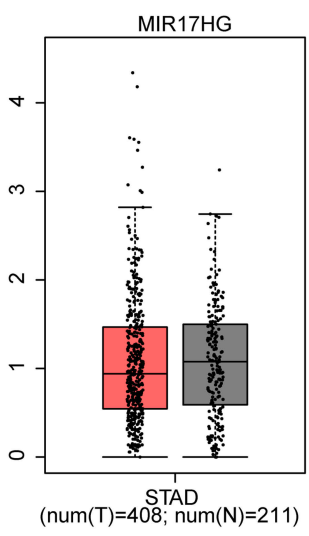
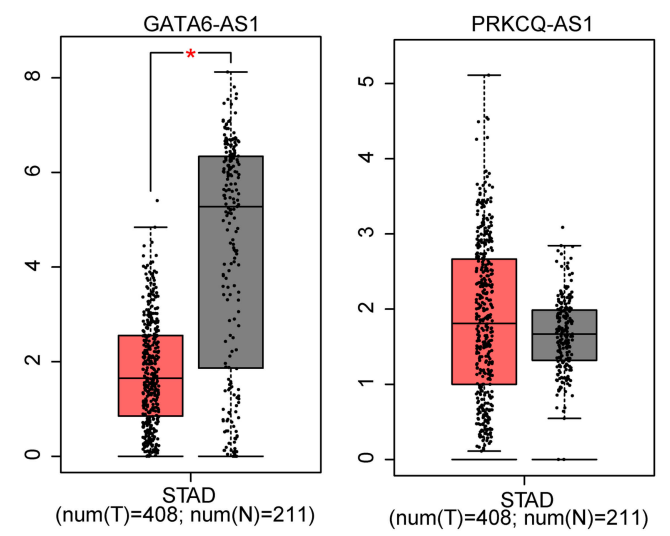

D

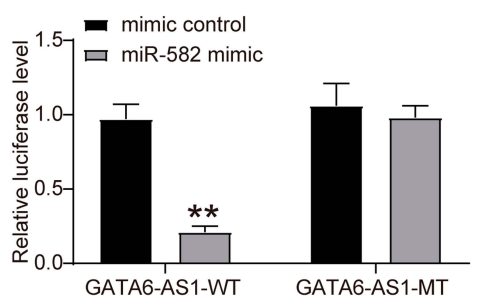

Figure 6 miR-582 binds specifically to GATA6-ASI. (A) The upstream targeting IncRNAs of miR-582 predicted by RNA22, StarBase and DIANA bioinformatics prediction websites. (B) NEATI, KCNQIOTI, MIRI7HG, GATA6-ASI and PRKCQ-ASI expression in gastric and normal tissues in the TCGA-STAD database of GEPIA websites. (C) RT-qPCR detection of GATA6-ASI expression in tumor and adjacent tissues of 45 patients with GC. (D) The binding relationship between miR-582 and GAGA6-ASI verified by dual luciferase assays. The data are displayed as the mean \pm SD of three independent experiments. Paired $t$-test (C) and two-way ANOVA and Tukey's multiple comparison test (D) was applied to determine statistical significance, $*_{p}<0.05, *_{p}<0.01$.

participated in the progression of cancers: oncogenes with promoted expression and tumor suppressors with decreased expression. ${ }^{21}$ The crucial regulatory function of miRNAs as diagnostic and therapeutic biomarkers in cancers is gradually documented by sequence-specific targeting oncogenes or tumor suppressors. ${ }^{22}$ In the present study, we screened the dysregulated miRNAs in GC by microarray analysis and verified that miR-582 was the most upregulated one in 45 cases of GC tissues by RTqPCR. Cell experiments showed that inhibition of miR582 could profoundly enhance E-cadherin expression and diminish $\mathrm{N}$-cadherin expression, thereby preventing GC cells from undergoing EMT and effectively attenuating the proliferation, migration and invasion abilities. Conversely, the PI3K specific agonist 740Y-P remarkably potentiated the proliferation, EMT, migration and invasion of GC cells. In addition, miR-582 was monitored to interact with GATA6-AS1 and FOXO3, respectively. Based on the abovementioned results, we believe that miR-582 acts as an oncogene in GC via the FOXO3-mediated PI3K/Akt/ Snail pathway.

With the intention of identifying vital miRNAs as GC therapeutic biomarkers in the study, five GC tissues were obtained from healthy persons and GC patients for microarray screening analysis, from which 30 miRNAs were screened out the plot the heatmap. Our RT-qPCR results further demonstrated that miR-582 was upregulated in the GC patients and closely correlated to metastasis. It has been suggested that miR-582 expression was significantly promoted, mediating induction of the $\mathrm{Wnt} / \beta$-catenin signaling pathway, and accelerating tumorigenesis and recurrence in non-small-cell lung carcinoma. $^{23}$ Besides, miR-582-5p was overexpressed in 
clinical tissues and colorectal cancer cells relative to adjacent tissues isolated from the same patients. ${ }^{24}$ Moreover, the proliferation, migration and invasion of colorectal cancer cells were demonstrated to be induced by overexpression of miR582, while miR-582 inhibition contributed to the opposite effect, indicating the oncogenic role of miR-582 in colorectal cancer. ${ }^{25}$ Through the online prediction databases, we found that FOXO3 was one of the unreported potential targets of miR-582. The dual luciferase activity reporter assay determined the targeting correlations between FOXO3, GATA6AS1 and miR-582. Upregulated GATA6-AS1 suppressed GC cell proliferation, invasion and migration. ${ }^{3-20}$ Nevertheless, the interaction between GATA6-AS1 and miRNAs has never been investigated before. In the present work, we identified the binding relationship between GATA6-AS1 and miR-582, while further investigation regarding this lncRNA and the downstream gene was necessary.

Additionally, the loss of FOXO3 interfered the transcription of its targets, leading to downstream responses that might accelerate tumorigenesis. ${ }^{26}$ Western blot analysis conducted by $\mathrm{He}$ et al showed that only FOXO3, rather than FOXO1, FOXO4 or FOXO6, was reduced in cancer regions of GC patients, ${ }^{18}$ which is in accordance with our current finding. FOXO3 is a well-known modulator of the $\mathrm{PI} 3 \mathrm{~K} / \mathrm{Akt}$ pathway in pancreatic cancer ${ }^{27}$ and hepatocellular carcinoma. ${ }^{28}$ Notably, tumor-associated macrophages facilitated the EMT process through the PI3K/Akt/Snail pathway, while impeding this axis with matrix metalloproteinase-9 proenzyme inhibitor repressed metastases in GC. ${ }^{29}$ Likewise, the PI3K/Akt/Snail pathway was probably linked to the enhancement of EMT through Aquaporin 3 in GC. ${ }^{30}$ In the current study, we verified that the PI3K specific agonist 740Y-P reversed the alteration of these cell activities mediated by miR-582. miR-582 could downregulate the expression of FOXO3 in GC cells, thereby inducing the activity of PI3K/Akt/Snail signaling transduction. Similar to our data, LINC02465 inhibition hampered the activation of the PI3K/Akt pathway, and the induction of this pathway through 740Y-P reversed the suppressive role of si-LINC02465 on the malignant phenotypes of GC cells. ${ }^{31}$ Many EMT-inducing transcription factors, including Snail have been linked to tumor invasion and metastasis. ${ }^{32}$ Therefore, repression of Akt or Snail counteracted the stimulative effect of family with sequence similarity 83 , member A overexpression in metastasis of non-small cell lung cancer. ${ }^{19}$ Consistent with our in vitro results, miR-582 downregulation also inhibited the growth and distant metastasis of mouse xenografts in vivo.
Immunohistochemical staining of the tissue sections disclosed that KI67 expression was profoundly reduced in mice treated with miR-582 downregulation.

\section{Conclusion}

In conclusion, we identified in the study that miR-582 was promoted in GC tissues and cells, which was associated with downregulated of GATA6-AS1. miR-582 could downregulate the expression of the target gene $\mathrm{FOXO} 3$ and increase the activity of $\mathrm{PI} 3 \mathrm{~K} / \mathrm{AKT} / \mathrm{Snail}$ signaling pathway, thus exerting a tumor-initiating effect on growth, invasion and metastasis of GC. Collectively, the GATA6-AS1/miR-582/ FOXO3/PI3K/AKT/Snail axis may have potential as a target for $\mathrm{GC}$ treatment and deserves further researches.

\section{Ethics Approval and Consent to Participate}

This study was permitted and supervised by the ethics committee of the Chinese PLA General Hospital. All the enrolled subjects provided written informed consents. The protocol was accepted by the Institutional Animal Care and Use Committee of the Chinese PLA General Hospital as well. All experiments involving animals were carried out following the Guide for the Care and Use of Laboratory animals published by the National Institutes of Health. Noteworthy efforts were made to curtail the number of animals and their sufferings.

\section{Author Contributions}

All authors made substantial contributions to conception and design, acquisition of data, or analysis and interpretation of data; took part in drafting the article or revising it critically for important intellectual content; gave final approval of the version to be published; and agree to be accountable for all aspects of the work.

\section{Disclosure}

The authors declare no potential conflicts of interest in this work.

\section{References}

1. Jemal A, Bray F, Center MM, Ferlay J, Ward E, Forman D. Global cancer statistics. CA Cancer J Clin. 2011;61(2):69-90. doi:10.3322/ caac. 20107

2. Yoon H, Kim N. Diagnosis and management of high risk group for gastric cancer. Gut Liver. 2015;9(1):5-17. doi:10.5009/gnl14118

3. Apicella M, Corso S, Giordano S. Targeted therapies for gastric cancer: failures and hopes from clinical trials. Oncotarget. 2017;8 (34):57654-57669. doi:10.18632/oncotarget.14825 
4. He FJ, Zhang P, Wang MJ, Chen Y, Zhuang W. Left armpit subcutaneous metastasis of gastric cancer: a case report. World J Clin Case. 2019;7(23):4137-4143. doi:10.12998/wjcc.v7.i23.4137

5. Zhang Y, Han T, Feng D, et al. Screening of non-invasive miRNA biomarker candidates for metastasis of gastric cancer by small RNA sequencing of plasma exosomes. Carcinogenesis. 2019. doi:10.1093/ carcin/bgz186

6. Hwang J, Min BH, Jang J, et al. MicroRNA expression profiles in gastric carcinogenesis. Sci Rep. 2018;8(1):14393. doi:10.1038/ s41598-018-32782-8

7. Song B, Long Y, Liu D, Zhang W, Liu C. MicroRNA-582 promotes tumorigenesis by targeting phosphatase and tensin homologue in colorectal cancer. Int J Mol Med. 2017;40(3):867-874. doi:10.3892/ ijmm.2017.3059

8. Liu Y, Jiang J, Wang X, Zhai F, Cheng X, Spencer J. miR-582-5p is upregulated in patients with active tuberculosis and inhibits apoptosis of monocytes by targeting FOXO1. PLoS One. 2013;8(10):e78381. doi:10.1371/journal.pone.0078381

9. Yang XB, Zhao JJ, Huang CY, et al. Decreased expression of the FOXO3a gene is associated with poor prognosis in primary gastric adenocarcinoma patients. PLoS One. 2013;8(10):e78158. doi:10.1371/journal.pone.0078158

10. Kim BK, Cheong JH, Im JY, et al. PI3K/AKT/beta-catenin signaling regulates vestigial-like 1 which predicts poor prognosis and enhances malignant phenotype in gastric cancer. Cancers (Basel). 2019;11 (12):1923. doi:10.3390/cancers11121923

11. Farhan M, Wang H, Gaur U, Little PJ, Xu J, Zheng W. FOXO signaling pathways as therapeutic targets in cancer. Int J Biol Sci. 2017;13(7):815-827. doi:10.7150/ijbs.20052

12. Yang Z, Guo X, Li G, Shi Y, Li L. Long noncoding RNAs as potential biomarkers in gastric cancer: opportunities and challenges. Cancer Lett. 2016;371(1):62-70. doi:10.1016/j.canlet.2015.11.011

13. Chen WJ, Tang RX, He RQ, et al. Clinical roles of the aberrantly expressed lncRNAs in lung squamous cell carcinoma: a study based on RNA-sequencing and microarray data mining. Oncotarget. 2017;8 (37):61282-61304. doi:10.18632/oncotarget.18058

14. Yang Y, Li Y, Hou Y, et al. Design, synthesis, and biological evaluation of 4-phenoxyquinoline derivatives as potent c-Met kinase inhibitor. Bioorg Med Chem Lett. 2019;29(23):126666. doi:10.1016/ j.bmcl.2019.126666

15. Feng RH, Zhu ZG, Li JF, et al. Inhibition of human telomerase in MKN-45 cell line by antisense hTR expression vector induces cell apoptosis and growth arrest. World $J$ Gastroenterol. 2002;8 (3):436-440. doi:10.3748/wjg.v8.i3.436

16. Chen Z, Ju H, Zhao T, et al. hsa_circ_0092306 targeting miR-197-3p promotes gastric cancer development by regulating PRKCB in MKN-45 cells. Mol Ther Nucleic Acids. 2019;18:617-626. doi:10.1016/j.omtn.2019.08.012

17. Finsterbusch K, Decker T, van Diest PJ, Focke CM. Luminal A versus B breast cancer: mammaTyper(R) mRNA versus immunohistochemical subtyping with emphasis on standardised Ki67-Labelling or Mitotic Activity Index based proliferation assessment. Histopathology. 2020;76(5):650-660.
18. He X, Zou K. MiRNA-96-5p contributed to the proliferation of gastric cancer cells by targeting FOXO3. J Biochem. 2020;167 (1):101-108.

19. Zhou F, Geng J, $\mathrm{Xu} \mathrm{S}$, et al. FAM83A signaling induces epithelial-mesenchymal transition by the PI3K/AKT/snail pathway in NSCLC. Aging (Albany NY). 2019;11(16):6069-6088. doi:10.18632/aging.102163

20. Li ZT, Zhang X, Wang DW, et al. Overexpressed lncRNA GATA6-AS1 inhibits LNM and EMT via FZD4 through the Wnt/ beta-catenin signaling pathway in GC. Mol Ther Nucleic Acids. 2020;19:827-840. doi:10.1016/j.omtn.2019.09.034

21. Asadi M, Shanehbandi D, Zafari V, Khaze V, Somi MH, Hashemzadeh S. Transcript level of MicroRNA processing elements in gastric cancer. $J$ Gastrointest Cancer. 2019;50(4):855-859. doi:10.1007/s12029-018-0154-8

22. Hosseinahli N, Aghapour M, Duijf PHG, Baradaran B. Treating cancer with microRNA replacement therapy: a literature review. J Cell Physiol. 2018;233(8):5574-5588. doi:10.1002/jcp.26514

23. Fang L, Cai J, Chen B, et al. Aberrantly expressed miR-582-3p maintains lung cancer stem cell-like traits by activating Wnt/beta-catenin signalling. Nat Commun. 2015;6:8640. doi:10.1038/ncomms9640

24. Shu Z, Chen L, Ding D. miR-582-5P induces colorectal cancer cell proliferation by targeting adenomatous polyposis coli. World J Surg Oncol. 2016;14(1):239. doi:10.1186/s12957-016-0984-4

25. Geng Y, Zheng X, Hu W, et al. Hsa_circ_0009361 acts as the sponge of miR-582 to suppress colorectal cancer progression by regulating APC2 expression. Clin Sci (Lond). 2019;133(10):1197-1213. doi:10.1042/CS20190286

26. Liu W, Song YY, Wang JY, Xiao H, Zhang Y, Luo B. Dysregulation of FOXO transcription factors in epstein-barr virus-associated gastric carcinoma. Virus Res. 2019;276:197808. doi:10.1016/j. virusres.2019.197808

27. Sun X, Kong L, Li B, Zhang Y, Yang H. Peroxiredoxin 1 silencing inhibited the growth and promoted apoptosis of pancreatic cancer cells via targeting FOXO3 gene. Cancer Manag Res. 2018;10:5019-5026. doi:10.2147/CMAR.S177243

28. Lu M, Hartmann D, Braren R, et al. Oncogenic Akt-FOXO3 loop favors tumor-promoting modes and enhances oxidative damage-associated hepatocellular carcinogenesis. BMC Cancer. 2019;19(1):887. doi:10.1186/s12885-019-6110-6

29. Liu L, Ye Y, Zhu X. MMP-9 secreted by tumor associated macrophages promoted gastric cancer metastasis through a PI3K/AKT/ Snail pathway. Biomed Pharmacother. 2019;117:109096. doi:10.1016/j.biopha.2019.109096

30. Chen J, Wang T, Zhou YC, et al. Aquaporin 3 promotes epithelial-mesenchymal transition in gastric cancer. $J$ Exp Clin Cancer Res. 2014;33:38. doi:10.1186/1756-9966-33-38

31. Han L, Hao Y, Wang J, Wang Z, Yang H, Wu X. Knockdown of LINC02465 suppresses gastric cancer cell growth and metastasis via PI3K/AKT pathway. Hum Gene Ther Clin Dev. 2019;30(1):19-28. doi:10.1089/humc.2018.177

32. Yang J, Weinberg RA. Epithelial-mesenchymal transition: at the crossroads of development and tumor metastasis. Dev Cell. 2008;14 (6):818-829. doi:10.1016/j.devcel.2008.05.009

\section{Publish your work in this journal}

Cancer Management and Research is an international, peer-reviewed open access journal focusing on cancer research and the optimal use of preventative and integrated treatment interventions to achieve improved outcomes, enhanced survival and quality of life for the cancer patient.
The manuscript management system is completely online and includes a very quick and fair peer-review system, which is all easy to use. Visit http://www.dovepress.com/testimonials.php to read real quotes from published authors 